\title{
La sociedad civil y la Convención de los Derechos de los Trabajadores Migratorios y sus Familias: alterna- tivas ante su débil consenso
}

\section{Daniela González Iza'}

Resumen. El desarrollo de normas internacionales de derechos humanos y de sus instituciones ha permitido cierto protagonismo de las organizaciones de la sociedad civil. El objetivo de este trabajo de investigación es analizar los alcances y límites de la sociedad civil en su interacción con el Comité para los Trabajadores Migratorios de las Naciones Unidas, tomando en cuenta que su instrumento normativo (la Convención de los Derechos de los Trabajadores Migratorios y sus Familias) es de los menos aceptados a nivel internacional. Por lo tanto, se evalúa hasta qué punto la falta de consenso internacional sobre la Convención puede representar una limitante en la relación de la sociedad civil con este Comité.

Palabras clave. Derechos humanos, Naciones Unidas, migración, sociedad civil, Convención de los Derechos de los Trabajadores Migratorios y sus Familias, Comité para los Trabajadores Migratorios.

Fecha de recepción: 4 de marzo de $2020^{2}$.

Fecha de admisión definitiva: 24 de abril de 2020.

\footnotetext{
1 Profesora del Tecnológico de Monterrey, Campus Santa Fe, Ciudad de México.

${ }^{2}$ Investigación ganadora del Premio Universidad Loyola a la Investigación en Desarrollo 2020.
} 
Civil society and the Convention on the Protection of the Rights of all Migrant Workers and Members of their Families: alternatives in the face of a weak consensus

Abstract: In the context of human rights, the development of international norms and institutions has allowed civil society organisations to play a certain role. The objective of this research is to analyse the achievements and the limits of civil society in its interaction with the United Nations Committee for Migrant Workers, taking into account the fact that the regulatory instrument (The Convention on the Rights of Migrant Workers and their Families) is one of the least accepted in the world. It assesses to which degree the lack of international consensus regarding the Convention might represent a limitation for the relationship between civil society and the Committee.

Key words: Human rights; civil society; migration; United Nations; Convention on the Protection of the Rights of Migrant Workers and Members of their Families; Committee on Migrant Workers.
La société civile et la Convention sur la Protection des Droits de tous les Travailleurs Migrants et des Membres de leur Famille: alternatives face à un faible consensus

Résumé: Le développement des normes internationales en matière de droits de I'homme et de leurs institutions a permis aux organisations de la société civile de jover un certain rôle. L'objectif de cette recherche est d'analyser la portée et les limites de la société civile dans son interaction avec le Comité des Nations Unies des Travailleurs Migrants, en tenant compte du fait que son instrument normatif (la Convention sur les Droits des Travailleurs Migrants et de leur Famille) est l'un des moins acceptés au niveau international. Ce travail évalue donc dans quelle mesure l'absence de consensus international sur la Convention représente une limitation dans les relations de la société civile arec ce Comité.

Mots clé: Droits de l'homme; Nations Unies; migration; société civil; Convention sur les droits des travailleurs migrants et ses familles; Comité des Travailleurs Migrants.

Desde la adopción de la Declaración Universal de Derechos Humanos, se han observado dos grandes fenómenos relacionados: el desarrollo internacional de normas de derechos humanos y la participación de actores no estatales en este proceso, como las organizaciones internacionales y organizaciones de la sociedad civil. El objetivo general de este trabajo de investigación es analizar el impacto de las acciones de la sociedad civil en el ámbito internacional de derechos humanos como estrategia de presión hacia los Estados.

¿Qué pasa cuando no hay suficiente consenso internacional sobre un determinado instrumento legal? Éste es el caso de la Convención para la Protección de los Derechos de los Trabajadores Migratorios y sus Familias (CMW, por sus siglas en inglés), la cual entró en vigor hace apenas un poco más de veinticinco años. Es una de las convenciones que no sólo tardó más de diez años en entrar en vigor, 
sino que es uno de los instrumentos de derechos humanos con más bajo índice de ratificaciones.

Aunado a que la gobernabilidad internacional en relación con la migración internacional es compleja, hay distintos intereses y razones por las cuales los Estados han sido reacios, no sólo a ratificar la CMW, sino a comprometerse de manera más profunda a otros instrumentos que ayudan a regular la gestión migratoria a nivel internacional. Por lo tanto, se evaluará hasta qué punto la falta de consenso internacional sobre la CMW puede representar una limitante en la relación de las Organizaciones No Gubernamentales (ONG) con el Comité para los Trabajadores Migratorios. Para ello, después de describir la relevancia y el contexto histórico de la adopción de la Convención, se analizará hasta qué punto hay o no un consenso internacional en la materia. De esta manera, se describirán los alcances y límites del Comité para los Trabajadores Migratorios, con especial énfasis en la relación que puede éste tener con la sociedad civil.

\section{La Convención de los Derechos de los Trabajadores Migra- torios y sus Familias}

La Convención de los Derechos de los Trabajadores Migratorios y sus Familias ha sido incorporada como parte del cuerpo normativo central del Sistema Universal de Derechos Humanos. Su relevancia no es mínima, considerando que es el primer instrumento global de protección de los derechos de los migrantes. Sin embargo, el régimen de protección de los derechos de los migrantes carece de apoyo internacional, tal como lo muestra la falta de ratificaciones de la CMW.

Hablar del fenómeno migratorio a nivel mundial es complejo debido a todos los temas que se relacionan entre sí. Aunque la libertad de tránsito es un derecho consagrado en la Declaración Universal de Derechos Humanos, corresponde a cada Estado la decisión sobre su política migratoria, lo cual ha creado intereses y expectativas divergentes en la gran mayoría de las veces.

Los datos recolectados sobre la migración en el mundo adolecen de imprecisión debido a la falta de homologación de estadísticas nacionales, a los rezagos en la realización de censos o a las dificultades para estimar la migración irregular; sin embargo, la información recabada indica que la migración internacional ha presentado características totalmente distintas a las del siglo veinte. De acuerdo con la Organización Internacional para las Migraciones, actualmente hay alrede- 
dor de 244 millones de migrantes en el mundo (3.3\% de la población mundial), 100 millones más que en 1990 . Alrededor del $72 \%$ de la población migrante se encuentra en edad productiva, $52 \%$ son hombres y el $48 \%$, mujeres (Organización Internacional para las Migraciones, 2018).

Si bien se conserva todavía una base de temporalidad, prácticamente se ha roto el esquema circular de la migración, aunado a la presencia de otros fenómenos relativamente nuevos en los patrones migratorios. Han incrementado la migración irregular y los delitos asociados a ésta (tales como el tráfico ilícito de inmigrantes y la trata de personas) ${ }^{3}$, se ha observado una feminización de los flujos migratorios (Sjöblo, Schiff, \& Morrison, 2008), así como la presencia de los llamados flujos mixtos, en donde es cada vez más complicado identificar entre solicitantes de asilo y migrantes (Taran, 2000, p. 87). A estas tendencias, habrá que sumar el arraigo de los discursos xenófobos en los países de destino, que se fortalecen ante la crisis económica y de seguridad. De acuerdo con Antonio Guterres, Secretario General de Naciones Unidas, "tristemente se reconoce que las narrativas políticas xenófobas sobre la migración se han extendido por todo el mundo" (Naciones Unidas, 2017, p. 4).

Hay que entender, asimismo, la vulnerabilidad específica que tiene la población migrante. Hay dos características que contribuyen a ella: su condición de extranjero y su condición laboral. Por un lado, la condición de extranjero se asocia con el desconocimiento de los países de destino, así como cierto escepticismo por parte de la sociedad receptora; por otro, los migrantes se insertan en los sectores menos favorecidos del mercado laboral (De Gutchteneire \& Pécoud, 2008, pp. 10-11). Se suman otras cuestiones, como la irregularidad, los delitos asociados a la migración y la explotación laboral. El enfoque de la gobernabilidad migratoria debería ser, entonces, la protección de los derechos de los migrantes.

Aunque el derecho al libre tránsito está reconocido en distintos instrumentos internacionales ${ }^{4}$, lo cierto es que la regulación migratoria reside exclusivamente en cada uno de los Estados. Los avances en la normatividad migratoria internacional se centran en aspectos relativos a derechos humanos, derecho laboral, crimen

\footnotetext{
${ }^{3}$ La Organización Internacional para las Migraciones explica la dificultad de recolección de datos relacionados con estos temas. Véase (Organización Internacional para las Migraciones, 2018).

${ }^{4}$ En la Declaración Universal de Derechos Humanos, la libertad de tránsito está reconocida en el artículo $13^{\circ}$. En el Pacto Internacional de Derechos Civiles y Políticos, este derecho está consagrado en el artículo $12^{\circ}$.
} 
transnacional organizado, refugio, protección consular, Derecho Internacional Humanitario y derecho marítimo (Unión Interparlamentaria, Organización Internacional del Trabajo y OACNUDH, 2015, pp. 40-43).

Dentro del sistema de Naciones Unidas, el tema migratorio se incluyó en la Conferencia Internacional sobre Desarrollo y Población (El Cairo, 1994). Asimismo, se han creado distintos mecanismos de cooperación internacional, como el Foro Global sobre Migración y Desarrollo, así como el Diálogo de Alto Nivel sobre Migración y Desarrollo ${ }^{5}$. En años más recientes, se han redoblado esfuerzos a mejorar la cooperación internacional migratoria desde distintas aristas, culminando con la reciente adopción del Pacto Mundial para la Migración Segura, Ordenada y Regular, en diciembre de $2018^{6}$. Actualmente, son más de veinte agencias de Naciones Unidas involucradas en el tema, siendo la más importante la Organización Internacional para las Migraciones, que se incorporó al sistema de la ONU hace un par de años.

No obstante, el fenómeno migratorio se ha debatido desde perspectivas centradas en los factores económicos y de seguridad, como lo ha apuntado Giuseppe Campesi (2012). Autores como Alejandro Canales (2015) indican que el debate también ha diferenciado las responsabilidades de países de destino y los de origen, cuando hay que entender a la migración desde un modelo de reproducción de clases sociales, del modelo económico y de la demográfica. Habrá que preguntarse los intereses que existen, tanto para cambiar o para conservar la estructuración del fenómeno migratorio actual y su gobernanza. En este sentido, es necesario el análisis sobre la dificultad en la adopción de normas migratorias y la falta de eficacia de la protección de los derechos humanos de los migrantes.

Dentro del Sistema Universal de Derechos Humanos, no había habido un documento específico para la protección de los migrantes en su condición de vulnerabilidad,

\footnotetext{
${ }^{5}$ Para analizar con más detalle la historia de la evolución del tema migratorio en la agenda de las Naciones Unidas, puede consultarse: (Slocum, 2017).

${ }^{6}$ El Pacto Mundial para la Migración Segura, Ordenada y Regular es un documento jurídicamente no vinculante donde se establecen veintitrés compromisos para promover una migración regular y segura. Entre estos objetivos, se encuentran: (1) enfrentar las causas estructurales de la emigración; (2) mejorar los procesos de admisión de migrantes; y (3) combatir los delitos asociados a la migración, como la trata de personas y el tráfico ilícito de inmigrantes. Otro de los principales puntos es evitar la detención de migrantes irregulares como primer recurso y mejorar los sistemas que permitan mejorar la protección de las poblaciones más vulnerables. Finalmente, se acuerdan crear mecanismos de seguimientos intergubernamental, como el Foro de Examen de la Migración Internacional (Naciones Unidas, 2018).
} 
aunque, como reconocen algunos autores, la CMW no nació para cubrir vacíos legales (Cano, 2010, p. 143). Algunos pasos previos ya se habían dado incluso antes de la creación formal de las Naciones Unidas, como lo fueron algunas convenciones adoptadas (y otras no ratificadas) en el seno de la Organización Internacional del Trabajo 7 . Asimismo, antes de la década de los setenta, era común la celebración de tratados bilaterales para regular el flujo de trabajadores migratorios, por lo que el periodo previo a la negociación de la CMW fue caracterizado por un aumento de preocupación sin normas homologadas y un establecimiento de estándares diferenciados; desde luego, un nuevo enfoque en la protección de los migrantes era necesario (Battistella, 2009, pp. 48-49).

El clima para negociar una convención para la protección de los migrantes era propicio en la década de los setenta. No solo el mundo atravesaba por la crisis energética y eventual crisis económica que impulsaban medidas migratorias más restrictivas, sino que también había nuevos países de destino en Medio Oriente. Algunos episodios relacionados con la vulnerabilidad de la migración irregular y la discriminación racial hicieron evidente esta necesidad, por parte de la ONU, de empezar un proceso de negociación de una nueva convención de derechos humanos que relacionara el tema laboral y migratorio ${ }^{8}$.

La legitimidad que había ganado la ONU con la adopción de la Carta Internacional de Derechos Humanos en 1966 impulsó la creación de un Grupo de Trabajo para

\footnotetext{
${ }^{7}$ Los convenios en vigor de la Organización Internacional del Trabajo son el Convenio no. 97 sobre trabajadores migrantes (1949), Convenio no. 118 sobre la igualdad de trato en seguridad social (1962), Convenio no. 143 sobre disposiciones complementarias sobre trabajadores migrantes (1975), Convenio no. 157 sobre la conservación de los derechos en materia de seguridad social (1982). Hay otros convenios que nunca entraron en vigor, como el Convenio no. 66 sobre los trabajadores migrantes (Organización Internacional del Trabajo, 2019).

${ }^{8}$ En 1972 fue conocida la noticia de un camión accidentado en Suiza que transportaba de manera clandestina personas provenientes de Mali que habían llegado desde Túnez y cuyo destino final era Francia. Fue el mismo ECOSOC (1706 (LIII)) quien solicitó que la Comisión de Derechos Humanos iniciara una investigación al respecto. La Subcomisión de Derechos Humanos encargó a Halima Warzazi, Relatora Especial de la Subcomisión de Prevención de Discriminaciones y Protección a las Minorías preparar un informe sobre la explotación de trabajadores que habían sido objeto de tráfico ilícito. El Informe Warzazi fue acogido durante la Conferencia Mundial para combatir el Racismo y la Discriminación Racial (Ginebra, 1978), haciendo un llamado a la adopción de un instrumento jurídico que protegiera a los trabajadores migratorios. El 20 de diciembre de 1978, en la Resolución 33/163, la Asamblea General de la ONU hizo el llamado oficial de explorar la oportunidad de negociar una convención internacional sobre los derechos de los migrantes. Finalmente, en 1979, la Asamblea General, en su Resolución 34/172, estableció un grupo de trabajo encargado de la redacción de una nueva convención (BATTISTELLA, 2009, pp. 50-53) (RODERA RANZ, 2014, pp. 144-147) (OACNUDH, 2006, p. 2).
} 
la redacción de la Convención. Aunque se habían adoptado algunos instrumentos jurídicos en el marco de la Organización Internacional del Trabajo (OIT), había cierto desencanto con esta organización, no sólo por su naturaleza tripartita entre gobiernos, empresas y sindicatos (Desmond, 2015, p. 43), sino por el texto del Convenio 143 adoptado en 1975, ya que desalentaba la migración temporal (De Gutchteneire \& Pécoud, 2008, p. 16). Además, se planteó la flexibilidad de adoptar un tratado internacional en el marco de la ONU que, a diferencia de los instrumentos adoptados en la OIT, permitieran la posibilidad de formular reservas (Rodera Ranz, 2014, p. 251).

De 1980 a 1990 el Grupo de Trabajo se reunió para redactar el texto de la Convención, lo cual hizo que, de una forma u otra, todos los países miembros de la ONU participaran en ello. Se identificaron diversos posicionamientos "grupales". México y Marruecos, apoyados por el Grupo de los 77, fueron los encargados de liderar el proyecto, ya que su interés se encontraba en proteger a sus connacionales en el exterior. Los países MESCA (países mediterráneos y escandinavos como Finlandia, Grecia, Italia, Portugal, España, Suecia y Noruega) tenían gobiernos con una posición socialdemócrata, lo cual facilitó una participación más activa. Finalmente, la OIT (quien, en un principio, deseaba ser el foro de negociación) también fue partícipe con el asesoramiento por parte de sus expertos (Desmond, 2015 , p. 43). Esta situación fomentó un mayor acercamiento de los expertos de la OIT en esta convención.

Hubo países que disentían de los principios expuestos en el texto. Alemania, Australia, Canadá, Dinamarca, Estados Unidos, Países Bajos y Japón llegaron a defender derecho de cada Estado a determinar los criterios de ingreso y regularización de extranjeros. Se identificaba que había países como Estados Unidos que no estarían de acuerdo en la ratificación desde un principio, aunque también se identificaba que había interés en dejar por sentado cierta normativa a nivel internacional (Rodera Ranz, 2014, pp. 268-269).

Fueron necesarios diez años para que el texto fuera finalmente adoptado. Battistella (2009, pp. 56-57) sugiere que el proceso de negociación se volvió tedioso debido a diversos factores, entre ellos el constante cambio de delegados (solamente los delegados de Marruecos y Finlandia los únicos que participaron ininterrumpidamente en todo el proceso) y los cambios acelerados en los procesos migratorios que se dieron a finales de los ochenta y el giro en los gobiernos europeos hacia políticas neoliberales. Asimismo, desde el inicio de la negociación, hubo temas que no sólo generaron desacuerdos, sino que estos impidieron llegar a consensos sobre un enfoque particular para el tratamiento de la migración internacional. 
Dichos desacuerdos se originaron desde la contradicción entre la soberanía del Estado y la protección de los derechos de las personas migrantes, lo cual quedaba reflejado en el debate sobre la no discriminación y el tratamiento de la migración irregular (Rodera Ranz, 2014, pp. 239-249).

Aunado a lo anterior, el proceso fue largo debido a las dificultades técnicas de la redacción de la convención. El método consistió en trabajar sobre un informe preliminar con un texto provisional de la Convención, tomando en cuenta otras normas internacionales, definiciones de términos, exposición de desacuerdos sobre algunos conceptos y redacción de versiones alternas al texto original de la Convención. El texto se adoptaría por consenso, no por votación (Rodera Ranz, 2014 , p. 260). El primer borrador preliminar se presentó en 1981, una versión reeditada (por parte de los países MESCA) en 1984. El texto definitivo se aprobó en junio de 1990. La Convención fue finalmente adoptada en la Asamblea General de la ONU el 18 de diciembre de 1990?.

\section{El consenso sobre la CMW}

La Convención se abrió a firma y a ratificación desde 1990. México fue el primer país en ratificar la Convención. Con veinte ratificaciones, entró en vigor en 2003, trece años desde su adopción, lo cual la convierte en uno de los instrumentos que más se ha tardado en entrar en vigor. Actualmente son 55 países parte y 13 países signatarios de la CMW, todos ellos, predominantemente expulsores de migrantes.

Aunque otros instrumentos jurídicos proveen principios aplicados a la comunidad migrante, la $\mathrm{CMW}$ es la primera convención en generar una protección más específica hacia los trabajadores migratorios, sin importar su condición jurídica en el país de destino. El objetivo de la Convención es establecer un mínimo de protección hacia los trabajadores migratorios, adoptar estándares que permitan evitar la explotación y delitos asociados, promoviendo así una armonización de estas normas con legislaciones nacionales. Para ello, la CMW define ciertos conceptos, como trabajador migratorio y familia, además que establece ciertas categorías de trabajadores, como los trabajadores fronterizos, temporales o por

\footnotetext{
9 Para una línea del tiempo sobre el proceso de adopción de la CMW puede consultarse: (Migrant Forum in Asia, 2019).
} 
cuenta propia $^{10}$. Finalmente, la CMW establece algunas diferencias entre la regularidad e irregularidad de los migrantes ${ }^{11}$.

La Convención se divide en nueve partes y 93 artículos: (1) Alcance y definiciones (artículos 1 a 6); (2) No discriminación en el reconocimiento de derechos (artículo 7); (3) Derechos humanos de todos los trabajadores migratorios y de sus familiares (artículos 8 a 35); (4) Otros derechos de los trabajadores migratorios y sus familiares que estén documentados o se encuentren en situación regular (artículos 36 a 56); (5) Disposiciones aplicables a categorías particulares de trabajadores migratorios y sus familiares (artículos 57 a 63); (6) Promoción de condiciones satisfactorias, equitativas, dignas y lícitas en relación con la migración internacional de los trabajadores y sus familiares (artículos 64 a 71); (7) Aplicación de la Convención (artículos 72 a 78); (8) Disposiciones generales (artículos 79 a 84); y (9) Disposiciones finales (artículos 85 a 93) (Naciones Unidas, 1990).

Los artículos de la Convención contemplan desde del principio de no discriminación ${ }^{12}$ hasta la descripción de derechos de migrantes documentados (incluso, se hace referencia implícita a la reunificación familiar en el artículo 44) ${ }^{13}$. Atención

${ }^{10}$ A efectos de la Convención, un trabajador migratorio es "toda persona que vaya a realizar, realice - haya realizado una actividad remunerada en un Estado del que no sea nacional" (Naciones Unidas, 1990, art. 2), mientras que los familiares son "las personas casadas con trabajadores migratorios o que mantengan con ellos una relación que, de conformidad con el derecho aplicable, produzca efectos equivalentes al matrimonio, así como los hijos a su cargo y otras personas a su cargo reconocidas como familiares por la legislación aplicable o por acuerdos bilaterales o multilaterales aplicables entre los Estados de que se trate" (NACIONES UNIDAS, 1990, art. 4).

${ }^{11}$ El artículo 5 de la Convención (NACIONES UNIDAS, 1990) establece la diferencia entre regularidad e irregularidad cuando los migrantes y sus familiares "han sido autorizados a ingresar, a permanecer y a ejercer una actividad remunerada en el Estado de empleo, de conformidad con las leyes de ese Estado y los acuerdos internacionales en que ese Estado sea parte".

${ }^{12}$ De acuerdo con el artículo 7 de la CMW, "Los Estados Partes se comprometerán, de conformidad con los instrumentos internacionales sobre derechos humanos, a respetar y asegurar a todos los trabajadores migratorios y sus familiares que se hallen dentro de su territorio o sometidos a su jurisdicción los derechos previstos en la presente Convención, sin distinción alguna por motivos de sexo, raza, color, idioma, religión o convicción, opinión política o de otra índole, origen nacional, étnico o social, nacionalidad, edad, situación económica, patrimonio, estado civil, nacimiento o cualquier otra condición" (NACIONES UNIDAS, 1990).

13 "Los Estados Partes tomarán las medidas que estimen apropiadas y entren en la esfera de su competencia para facilitar la reunión de los trabajadores migratorios con sus cónyuges o con aquellas personas que mantengan con el trabajador migratorio una relación que, de conformidad con el derecho 
especial merecen los artículos que hablan de los derechos de todos los trabajadores migratorios, independientemente de su situación de regularidad (artículos 8 al 35), donde sobresalen la prohibición de la expulsión colectiva o sin el debido proceso judicial, la obligación de otorgar asistencia consular, y el deber de garantizar trato igualitario en el trabajo, así como en servicios de seguridad social, médicos y educativos.

La Convención establece obligaciones de adoptar políticas migratorias, tanto para países de origen, como de tránsito, tratando de equilibrarlo con el respeto a las legislaciones nacionales. Por ejemplo, se menciona la importancia de la cooperación internacional en materia migratoria ${ }^{14}$, así como las funciones que desempeñará el Comité de Trabajadores

Migratorios en el monitoreo de las obligaciones contraídas por los Estados parte (Naciones Unidas, 1990, arts. 72-77). Se establece, además, la obligación de los Estados de proveer toda la información, tanto a empleadores como a trabajadores, con el fin de evitar la explotación laboral y la migración irregular (Naciones Unidas, 1990 , arts. 68 y 69). Finalmente, la Convención es clara en cuanto a los procesos de cada país en cuestiones de admisión de extranjeros (Naciones Unidas, 1990, art. 79), regularización (Naciones Unidas, 1990, art. 35) o en el reconocimiento de participación política, por ejemplo (Naciones Unidas, 1990, art. 41).

Taran (2000, pp. 89-90) explicita las razones por las cuales la Convención es relevante. En primer lugar, la Convención reconoce al migrante más allá de su condición laboral, lo cual implica vulnerabilidad en las sociedades de acogida que no son tratadas adecuadamente. Asimismo, provee, por primera vez, definiciones, categorías y derechos relacionados con el fenómeno migratorio, tanto irregular como regular, con el fin de establecer mínimos estándares internacionales de protección. Finalmente, la Convención se involucra en cuestiones como la prevención del tráfico ilícito de inmigrantes.

aplicable, produzca efectos equivalentes al matrimonio, al igual que con sus hijos solteros menores de edad que estén a su cargo" (NACIONES UNIDAS, 1990, art. 44).

${ }^{14}$ El artículo 67 de la CMW establece que "los Estados partes interesados cooperarán de la manera que resulte apropiada en la adopción de medidas relativas al regreso ordenado de los trabajadores migratorios y sus familiares al Estado de origen cuando decidan regresar, cuando expire su permiso de residencia o empleo, o cuando se encuentren en situación irregular en el Estado de empleo. Por lo que respecta a los trabajadores migratorios y sus familiares que se encuentren en situación regular, los Estados Partes interesados cooperarán de la manera que resulte apropiada, en las condiciones convenidas por esos Estados, con miras a fomentar condiciones económicas adecuadas para su reasentamiento." 
Aunado a que la Convención es parte de los tratados centrales del Sistema Universal de Derechos Humanos, es uno de los grandes intentos por promover, desde la perspectiva de los derechos humanos, una mejor gobernabilidad del fenómeno migratorio internacional. De hecho, los derechos contenidos en la Convención coinciden con otros instrumentos jurídicos, aunque también la Convención extiende algunos de ellos, crea nuevos 0 , incluso, establece ciertas regulaciones (Nafziger \& Bartel, 1991, pp. 781-784).

¿En qué medida hubo entonces un consenso internacional cobre la CMW? Analizando las ratificaciones de esta convención, podemos decir que es una de las convenciones con menor número de Estados parte. Hasta 1998, la Convención contaba con nueve ratificaciones, y actualmente, la Convención cuenta con 55 Estados parte y 39 Estados signatarios (trece de ellos no la han ratificado), siendo uno de los tratados centrales del Sistema Universal de Derechos Humanos menos apoyados a nivel internacional. Como se mencionó anteriormente, la Convención se abrió a firma en 1990, siendo México y Marruecos los primeros países en firmar. La CMW logró entrar en vigor el $1^{\circ}$ de julio de 2003 y ese año la Convención alcanzó las 24 ratificaciones. El avance más importante se dio de 2004 a 2005, al pasar de 27 Estados parte a 34 .

\section{Consenso internacional sobre la CMW}

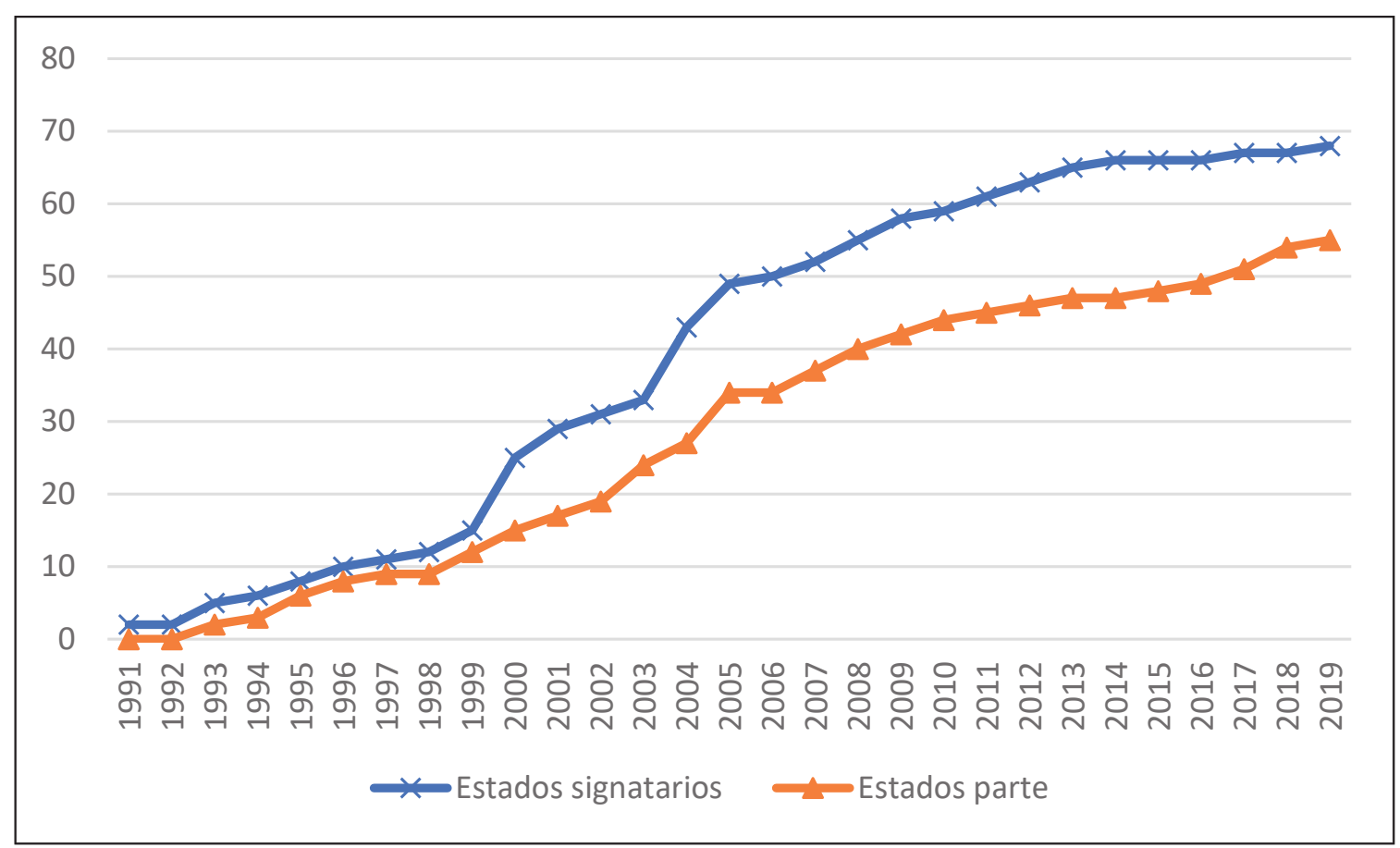

Fuente: United Nations Treaty Collection, 2019. 
Analizando los periodos de tiempo entre las firmas y las ratificaciones, el $46 \%$ de los Estados parte de la Convención se adhirieron de forma inmediata y el 18\% tardó de uno a cuatro años en ratificar. Solamente el $7 \%$ de los Estados parte tardaron más de diez años entre su firma y su ratificación. Esto puede darnos un indicio que los procesos de aprobación a nivel interno han sido relativamente rápidos en algunos países, facilitando así la ratificación de este instrumento.

La Convención no interpone ninguna restricción a la formulación de reservas, siempre y cuando no vayan en contra del objeto y fin del tratado (Naciones Unidas, 1990, art. 91). Considerando el número de artículos, hay que hacer la observación que este instrumento no adolece de tantas reservas o declaraciones interpretativas, como otros tratados internacionales de derechos humanos. Actualmente, la CMW cuenta con apenas 12 reservas a diez artículos y 26 declaraciones interpretativas a doce artículos ${ }^{15}$. De los 55 Estados parte, siete son los que han adoptado alguna reserva, ya sea a todo el artículo o a algún párrafo específico del mismo (Argelia, Chile, Colombia, Egipto, Fiii, Nicaragua, Turquía y Uganda). El país que ha hecho más reservas es Colombia, quien hizo reservas a los artículos 15, 46 y 47.

\section{Años de diferencia entre la firma y la ratificación de la CMW}

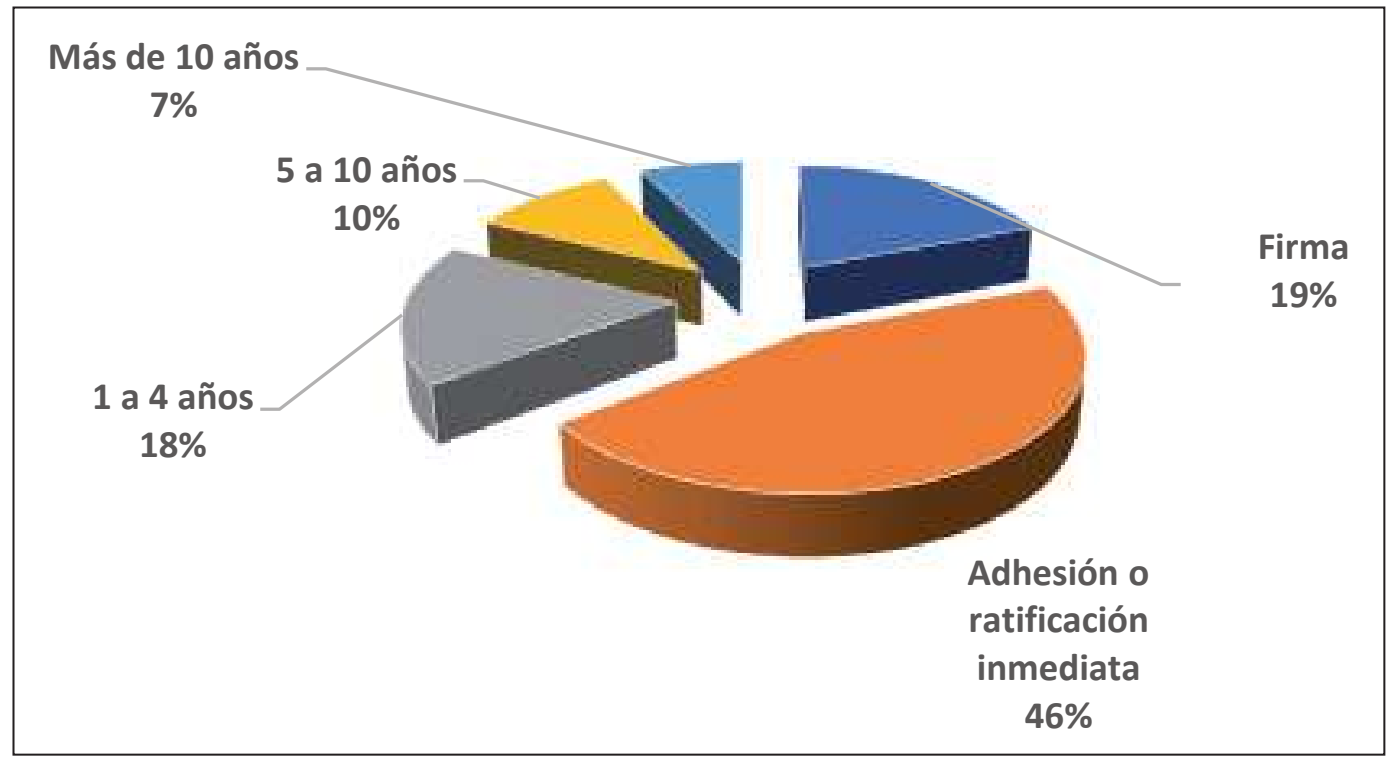

Fuente: United Nations Treaty Collection, 2019.

${ }^{15}$ Los artículos que tienen reservas son los artículos $4,15,18,22,40,42,46,47,48$ y 92 . Los artículos que han sido objeto de alguna declaración interpretativa son los artículos $8,15,45,46,47,48,49$, 54, 61, 76, 77 y 92 (United Nations Treaty Collection, 2019). 
Aunque puede decirse que las reservas son aisladas (como por ejemplo, la que hizo Egipto al artículo $4^{\circ}$ que define el concepto de "familia", la reserva que hizo Chile al artículo 22, punto 5, sobre las indemnizaciones en caso de revocación de expulsión, la reserva al artículo 40 por parte de Turquía sobre el derecho a formar sindicatos o la reserva que hizo Nicaragua al artículo 42, punto 3, sobre los derechos políticos de los trabajadores migratorios), el artículo que tiene más reservas (una por parte de Egipto y otra por parte de Uganda) es el 18, el cual habla del trato igualitario ante cortes nacionales ${ }^{16}$. Por otro lado, aunque el efecto jurídico de una reserva es distinto a la de una declaración interpretativa, hay artículos que han sido objeto de ambas; por ejemplo, el artículo 15, los artículos 46,47 y $48{ }^{17}$.

Caben destacar las declaraciones realizadas a los artículos 76, 77 y 92 . En el artículo 77, los Estados deben hacer una declaración para reconocer la competencia del Comité para recibir y examinar comunicaciones individuales. En este caso, se tienen registradas seis declaraciones aceptando la competencia del Comité (Ecuador, El Salvador, Guatemala, México y Uruguay). Caso contrario es el artículo 76, que permitiría que el Comité reciba comunicaciones de un Estado contra otro Estado, que solo ha recibido la aceptación por parte de Ecuador, el Salvador y Guinea Bissau. En el caso del artículo 92, que habla del procedimiento en caso de disputa entre dos países parte de la Convención, ha recibido dos reservas (por parte de Argelia y Fiii) y cinco declaraciones (por parte de Argentina, El Salvador, Guinea Bissau, Marruecos y Venezuela) cuyo contenido es similar a las reservas formuladas a este artículo (United Nations Treaty Collection, 2019).

Si bien la Convención no ha sido objeto de múltiples reservas, la observación recae en el bajo índice de ratificaciones. ¿Esta situación nos podría dar un indicio sobre el bajo consenso internacional sobre la Convención? Diversos autores analizan las razones por las cuales ha habido una baja participación de los países para comprometerse de manera más profunda en la CMW.

\footnotetext{
${ }^{16}$ Egipto formuló una reserva al punto 6 del artículo 18. En el caso de Uganda, la reserva fue al artículo 18, punto 3(d), explicando que no "puede garantizar siempre la asistencia legal gratuita" (United Nations Treaty Collection, 2019).

${ }^{17}$ El artículo 15 habla de las condiciones en las expropiaciones de bienes a los trabajadores migratorios. Actualmente, Colombia formuló una reserva al respecto y Turquía, una declaración interpretativa. Los artículos 46,47 y 48 hablan de distintas exenciones de impuestos a los trabajadores migratorios y sus familiares, con el objetivo de evitar doble tributación. Colombia hizo reservas a los artículos 46 y 47, bajo el entendido que "Colombia sostiene el derecho de promulgar sus propias leyes respecto a impuestos." Por otro lado, Chile adoptó una reserva al artículo 48 punto 2 (United Nations Treaty Collection, 2019).
} 
Por ejemplo, Nafizger y Bartel (1991, p. 787) apuntan que el problema se encuentra en la misma Convención. Al tomar en cuenta diversos temas al mismo tiempo hacia distintos colectivos y con un lenguaje específico, puede provocar cierto entorpecimiento en la aplicación de otros instrumentos. Por lo tanto, la Convención puede considerarse ambiciosa en ese sentido, lo cual ha repercutido negativamente en el número de ratificaciones a nivel internacional.

Un segundo factor ha sido la falta de información alrededor de la Convención. De acuerdo con Guchteneire y Pécoud (2009, pp. 12-17), no sólo hubo una ola de desinformación respecto al contenido de la Convención (llegándola a considerar "el secreto mejor guardado de la ONU") ${ }^{18}$, lo cual generó confusión sobre la supuesta "pérdida de soberanía" (por ejemplo, en Alemania o en Italia), sino que también los países que ya tenían normas migratorias no consideraban "necesario" ser parte de ella (por ejemplo, Bélgica). Incluso, otros países (por ejemplo, en Asia) han aludido a la falta de recursos técnicos o presupuestarios para cumplir con la Convención. Una manera en la que se intentó acelerar el proceso de ratificación fue, precisamente, a través de campañas de concientización sobre el contenido de la Convención, siendo el esfuerzo más importante la Campaña Global por la Ratificación de la CMW en 1998, además que la ONU no desistió en el tema (Battistella, 2009, pp. 59-61).

El gran obstáculo continúa siendo la falta de voluntad por parte de los países de destino, principalmente. En países como Alemania, Canadá, Francia, España o Gran Bretaña, el debate en torno a aceptar la Convención ha tocado algunos temas sensibles, tales como cuestiones de integración cultural o el tratamiento de la migración irregular (De Guchteneire \& Pécoud, 2009, pp. 18-22). En un estudio realizado por Jorge Bustamante (2002, pp. 352-353), hay grupos poderosos muy concretos a nivel nacional que se benefician de la manera en cómo se ha llevado a cabo la gestión migratoria. Estos intereses internos se vuelven más relevantes, en la medida que haya un contexto poco favorable, como crisis económicas, por ejemplo (Ruhs, 2013, pp. 33-34).

Ahora, hay que tomar en cuenta la coyuntura internacional de cuando la CMW fue adoptada y la actual. Si bien hubo una división muy clara entre los intereses de los países de destino y los países de origen en ser parte de la Convención (Battistella, 2009, pp. 60-61), la realidad es que el fenómeno se ha vuelto más complejo. No

\footnotetext{
${ }^{18}$ TARAN (2000, p. 95) explica que la falta de promoción del texto de la Convención fue también error de Naciones Unidas, ya que era difícil obtener el texto, hasta 1996, cuando se publicaron materiales al respecto.
} 
es posible identificar canales claros en los patrones migratorios, profundizando los intereses de dejar de lado la Convención por parte de los países que no son parte ${ }^{19}$.

La situación por la que atraviesa la $\mathrm{CMW}$ se enmarca en un escenario aún más amplio sobre los desacuerdos que existen en la gobernabilidad internacional de la migración, cuestión que logra percibirse con el bajo índice de ratificaciones de otros instrumentos similares ${ }^{20}$. Se han adoptado documentos que tratan temas más específicos, como la Convención de las Naciones Unidas contra la Delincuencia Organizada Transnacional y sus respectivos Protocolos, que generan todavía mayor animadversión hacia la ratificación de la Convención ${ }^{21}$. Finalmente, autores como Pécoud (2017, pp. 38-39) observan el aumento de mecanismos regionales o bilaterales que excluyen de cierta manera los estándares adoptados en la ONU.

Es más, el nuevo Pacto Mundial para la Migración hace escasa referencia directa a la Convención ${ }^{22}$. El Pacto Mundial toca, de manera muy general, desde un enfoque muy tradicional sin caer en definiciones, la prevención de la migración irregular ${ }^{23}$,

19 En los países de destino, la no ratificación de la Convención constituye un símbolo del reconocimiento
de los derechos de los migrantes, independientemente de su estatus legal, por lo que se encuentra
resistencia ante los sentimientos anti migrantes. En el caso de los países de origen, la ratificación de la
Convención es una estrategia multilateral de protección a sus ciudadanos en el exterior; sin embargo,
esta división también se enmarca en un continuo debate Norte - Sur (Pécoud, 2017, pp. 36-37).

20 Por ejemplo, los Convenios 97 y 143 de la OIT cuentan con 50 y 24 ratificaciones, respectivamente. En el caso del Convenio 189 de la OIT Convenio sobre las trabajadoras y los trabajadores domésticos (que entró en vigor en 2013) cuenta apenas con 29 ratificaciones (Organización Internacional del Trabajo, 2019). Se estima que alrededor de 87 de países son parte de, por lo menos, una convención de la Organización Internacional del Trabajo relativo a la migración (Unión Interparlamentaria, Organización Internacional del Trabajo y OACNUDH, 2015, pp. 53-55).

${ }^{21}$ La Convención de las Naciones Unidas contra la Delincuencia Organizada Transnacional (Convención de Palermo) fue adoptada el 15 de noviembre del año 2000 y entró en vigor el 29 de septiembre de 2003. Cuenta con 190 Estados parte a julio de 2018. Esta Convención es relevante porque, además de definir conceptos importantes en torno a crimen transnacional organizado, tiene tres protocolos que ayudan a combatir estos crímenes: (1) el Protocolo para prevenir, suprimir y castigar la trata de personas, especialmente mujeres y niños; (2) el Protocolo contra el tráfico ilícito de migrantes por tierra, mar y aire; y (3) el Protocolo contra la fabricación y el tráfico ilícitos de armas de fuego, sus piezas y componentes y municiones (UNODC, 2019).

${ }^{22}$ De hecho, el Comité se pronunció al respecto con profunda preocupación, mandando cartas a los co facilitadores de la redacción del Pacto Mundial para la Migración (las representaciones de México y Suiza) (Comité CMW, 2018, p. 4).

${ }^{23}$ Se reconoce que cada país tendrá que hacer una distinción entre migración regular e irregular, 
compromisos de acuerdo con la realidad migratoria de cada país ${ }^{24}$, y el respeto a los derechos humanos y garantía de una vida digna de los migrantes. Actualmente, más de una decena de países se han rehusado a adoptar los compromisos incluidos en el Pacto Mundial (a pesar de no ser jurídicamente vinculante), aludiendo a la soberanía nacional, la seguridad y el interés de Estado ${ }^{25}$. Por lo tanto, la compleja realidad por la que atraviesan las dinámicas migratorias hace imposible pensar que el consenso internacional sobre la CMW vaya en aumento.

Vale la pena matizar estos argumentos. En primer lugar, hay que recordar que la redacción del contenido de la Convención fue también producto de la participación de países que no eran solo expulsores; de hecho, países europeos (como el grupo MESCA) estuvieron involucrados en el proyecto. Battistella $(2009$, p. 55) y $(2015$, p. 44) defienden la idea que el texto de la Convención es, sorpresivamente, un texto europeo, si se analiza la manera en que participaron los países del grupo MESCA; incluso, países que no estuvieron de acuerdo con la adopción de la Convención evitaron entorpecer el proceso. El resultado fue la adopción de estándares mínimos de protección que permitan un equilibrio entre la garantía de derechos a la población migrante y el respeto a la administración migratoria en cada país.

Se reconoce que el valor añadido de la Convención radica en establecer un sistema de protección integral en la entrada y estadía de migrantes, así como de derechos de salud y protección social que va más allá de las premisas que establecer otros tratados de derechos humanos (Georgopoulou, Schrempf, \& Venturi, 2017, pp. 130-142). Autores como Lesley Wexler reconocen el papel no tradicional de estas normas que no dependen de la ratificación: (1) proveen material para otras iniciativas multilaterales, como los Procesos Regionales de Consulta; (2) proporcionan ejemplos de mejores prácticas; y (3) son parte de un marco discursivo (Wexler, 2007, pp. 376-396). Entonces, ¿̇hay esperanza ante la ausencia de ratificaciones de la Convención?

tomando en cuenta las particularidades nacionales, normativas, y requerimientos de entrada (Naciones Unidas, 2018).

${ }^{24}$ Entre otros, se habla del trabajo decente, de la relación entre migración irregular y vulnerabilidad, o del combate al crimen transnacional organizado (Naciones Unidas, 2018).

${ }^{25}$ Estados Unidos argumentó desde un principio que no sería parte del Pacto Mundial. Países como Austria, Hungría, Eslovaquia, Australia y Polonia han argumentado que los compromisos adoptados en el Pacto atentan contra los intereses nacionales y su autonomía para tomar decisiones sobre asuntos migratorios. En Alemania y en Italia, la adopción del Pacto fue objeto de profundos debates internos (Carrera, Lannoo, Stefan \& Vosyliūtè, 2018, pp. 3-4). 
Algunos autores han analizado cómo se presentan e interpretan las normas contenidas en la Convención en otros instrumentos jurídicos del Sistema Universal de Derechos Humanos (Slinckx, 2009, pp. 122-149) (Grant \& Lyon, 2017, pp. 104-108) (OACNUDH, 2006, pp. 14-18). Asimismo, hay estudios que intentan monitorear las políticas migratorias en países que no son parte de la Convención, con el fin de minimizar los efectos del poco consenso que tiene este instrumento por ejemplo, programas de prevención, políticas de monitoreo de posibles actos de abuso por parte de empleadores y reclutadores, y servicios de reparación (Martin \& Abimourched, 2009, pp. 125-133). Si bien la ratificación de la CMW puede evitar que se tomen medidas que resulten ineficientes o que sean totalmente provisionales, hay que reconocer que existe cierta compensación ante la falta de consenso internacional de este instrumento.

Finalmente, hay que tomar en cuenta los beneficios que podrían suponer las nuevas realidades coyunturales. Por ejemplo, Antoine Pécoud (2017, p. 31) se muestra optimista al mostrar que son cada vez más países que se enfrentan a una realidad donde la inmigración, emigración y transmigración se inserta en sus sociedades, por lo que la CMW puede ser de ayuda. Esta situación puede ser impulsada por la presión que puedan ejercer otros actores como la sociedad civil en su interacción con el Comité de Trabajadores Migratorios.

\section{Monitoreando la CMW: el Comité de Trabajadores Migratorios}

El órgano encargado de monitorear los avances de los países parte de la CMW es el Comité de los Trabajadores Migratorios, cuyas funciones se encuentran descritas en la Parte VII de la Convención (artículos 72 a 78). El Comité es relativamente nuevo, ya que empezó sus labores en marzo 2004 con apenas diez expertos independientes, electos a título personal en votación secreta de los Estados parte para periodos de cuatro años. Al alcanzar las 41 ratificaciones, el número de expertos del Comité incrementó a catorce (a partir de 2010).

Las principales funciones del Comité son básicamente las mismas que las de los ocho órganos creados en virtud de tratados. De acuerdo con el artículo 73 de la CMW, los Estados parte tienen la obligación de presentar informes periódicos sobre los avances en los compromisos adoptados, de acuerdo con las directrices que publique el Comité al respecto. Los Estados deben presentar un informe inicial al año de haber ratificado la Convención y los informes subsecuentes cada cinco 
años. Para ello, el Comité publicó una serie de directrices generales, además que periódicamente publica una lista de temas a tratar para cada informe que deba ser presentado 26 . Durante la revisión, el Comité invita a los Estados a formar parte del examen en sesiones públicas, con el fin de respetar el principio de "diálogo constructivo" (Asamblea General de Naciones Unidas, 2014). En reunión privada, los expertos del Comité elaboran sus observaciones finales, que serán publicadas y atendidas en el siguiente informe periódico.

En 2017, el Comité recibió un promedio de seis informes periódicos anualmente. Solamente el $8 \%$ de los Estados parte de la CMW tenían algún informe inicial atrasado, mientras que el 10\% de los Estados parte tenían algún atrasado en la entrega de informes (solamente uno tiene más de cinco años de atraso). En promedio, este Comité revisa seis informes, dedicando alrededor de cuatro semanas al año distribuidas en dos sesiones, en Ginebra. A diciembre de 2017, solamente el $8 \%$ de los informes recibidos se consideraban pendientes de revisión, un $14 \%$ más que en 2015 (Naciones Unidas, 2018, pp. 8-12).

El Comité de Trabajadores Migratorios tiene un índice bajo de informes atrasados. Aunque esto se deba, primordialmente, al bajo índice de ratificaciones de la CMW, también hay que tomar en cuenta los recientes esfuerzos que los expertos del Comité, en cooperación con los otros mecanismos convencionales, han realizado para eficientizar los procesos de entrega y revisión de los informes periódicos de acuerdo con la resolución de la Asamblea General de la ONU (A/68/268) (Naciones Unidas, 2018).

Asimismo, este Comité tiene contempladas en la Convención algunas funciones exclusivas como las demandas entre Estados y las comunicaciones individuales (Naciones Unidas, 1990, arts. 76 y 77). Para ello, es los Estados parte deben realizar una declaración aceptando estas competencias, acorde a estos artículos. Una vez que más de diez Estados acepten dicha competencia, el Comité estará facultado para ello.

Ahora, los expertos del Comité también se reúnen para otros fines, lo cual ha hecho desde el inicio de sus funciones (Edelenbos, 2009, pp. 112-118). Recientemente, el Comité ha organizado reuniones para adoptar observaciones generales sobre

${ }^{26}$ Las directrices para los informes iniciales mencionan que los Estados parte deben presentar el Documento Básico Común a todos los Comités (Naciones Unidas, 2005). En el caso de los informes periódicos, estos responderán a una lista de cuestiones previamente publicada por el Comité (Comité CMW, 2008). 
alguna temática en específico que, incluso, han sido conjuntas con otros comités ${ }^{27}$, posiciones oficiales ${ }^{28}$, así como declaraciones y otros comunicados ${ }^{29}$. La relevancia de estos posicionamientos reside en que se da una mayor visibilidad a ciertos temas que, incluso, no están expresamente incluidos en la Convención ${ }^{30}$.

El Comité sostiene reuniones con otros actores dentro del Sistema ONU len particular con la Organización Internacional para las Migraciones, UNICEF y ONU Mujeres), y fuera de él (como la OIT), con el fin de promover la Convención. En el último informe anual del Comité CMW (2018, pp. 4-6) algunas de las acciones que realizaron los expertos tuvieron que ver con la reunión con otras organizaciones internacionales, asesoramiento a Estados parte sobre la entrega de informes, colaboración en diversas publicaciones, y participación en foros académicos y de la sociedad civil. Finalmente, el Comité también se ha dado a la tarea de promover la ratificación de la Convención, participando activamente en el Comité Directivo Internacional de la Campaña Mundial por la Ratificación de la Convención sobre los Derechos de los Trabajadores Migratorios, el cual está integrado por representantes de organizaciones internacionales, ONG, organizaciones religiosas y laborales ${ }^{31}$.

\footnotetext{
${ }^{27}$ Las observaciones generales publicados por el Comité de Trabajadores Migratorios son: (1) Observación General ${ }^{\circ} 1$ sobre trabajadores domésticos migratorios (2011); (2) Observación General $\mathrm{n}^{\circ} 2$ sobre los derechos de los trabajadores migratorios en situación irregular y de sus familiares (2013); (3) Observación general conjunta $n^{\circ} 3$ (2017) del Comité de Protección de los Derechos de Todos los Trabajadores Migratorios y de sus Familiares y $n^{\circ} 22$ (2017) del Comité de los Derechos del Niño sobre los principios generales relativos a los derechos humanos de los niños en el contexto de la migración internacional; y (4) Observación General conjunta n 4 (2017) del Comité de Protección de los Derechos de Todos los Trabajadores Migratorios y de sus Familiares y n 23 (2017) del Comité de los Derechos del Niño sobre las obligaciones de los Estados relativas a los derechos humanos de los niños en el contexto de la migración internacional en los países de origen, tránsito, destino y retorno (OACNUDH, 2019). Actualmente, existe el proyecto de una Observación General sobre los derechos de los migrantes a la libertad y la libertad de una detención arbitraria.
}

${ }^{28}$ Por ejemplo, en el caso del Diálogo de Alto Nivel de Naciones Unidas sobre los Objetivos de Desarrollo Sostenible (Comité CMW, 2018) o del Reporte del Secretario General de la ONU sobre el Pacto Mundial para la Migración (Comité CMW, 2017).

29 Por ejemplo, destacan los comunicados conjuntos con el Relator Especial sobre los Derechos Humanos de los Migrantes en relación con el Día Internacional del Migrante (18 de diciembre) (OACNUDH, 2018).

${ }^{30}$ En un análisis sobre la funcionalidad de la $\mathrm{CMW}$ en materia de detenciones arbitrarias de migrantes, Mariette Grange (2017, pp. 95-98) opina que una observación general sobre el tema podría ser muy útil. Incluso, se propone adoptar el método de las observaciones generales en conjunto con otros mecanismos, considerando las limitaciones de la CMW.

${ }^{31}$ Las organizaciones parte de este esfuerzo son: December 18, FIDH, Global Migration Policy As- 
Por lo tanto, las tareas del Comité CMW van más allá de la revisión de informes periódicos. Las funciones "oficiales" han ocupado solo una parte en el tiempo que los expertos dedican en sus sesiones del Comité, siendo cada vez más importantes otras tareas como la promoción de la Convención y las campañas dedicadas a su ratificación. Por supuesto, esta situación puede plantear retos en cuanto a las cuestiones logísticas y presupuestarias; sin embargo, también plantea la relevancia que otros actores están presentando. Particularmente, ¿̇la interacción que el Comité sostiene con la sociedad civil nos presenta algunos escenarios prometedores ante la falta de consenso de la Convención? ¿¿Qué estrategias se han tomado ante la debilidad de la CMW?

\section{El Comité de los Trabajadores Migratorios y sociedad civil: estrategias ante un débil consenso}

Al igual que varios de los mecanismos convencionales, la relación del Comité de Trabajadores Migratorios y la sociedad civil ha ido intensificándose al paso el tiempo. En la CMW se menciona la participación de la sociedad civil de manera indirecta ${ }^{32}$. La participación de la sociedad civil en los distintos procesos del Comité quedaron por sentadas, tanto en sus Reglas de Procedimiento, como en las Directrices para la presentación de informes ${ }^{33}$.

sociates, Human Rights Watch, International Catholic Migration Commission, Migran Forum in Asia, Migrant Rights International, la Internacional de Servicios Público, el Consejo Mundial de Iglesias y la Confederación Sindical Internacional. Entre las agencias internacionales que forman parte de este proyecto, se encuentran: la Organización Internacional del Trabajo, Organización Internacional para las Migraciones, la Confederación Sindical Internacional, la Oficina del Alto Comisionado de Naciones Unidas para los Derechos Humanos, y la UNESCO (Steering Committee on Global Campaign for Ratification of the Convention on the Rights of Migrants, 2019).

32 En el artículo 74 de la CMW, se establece que "el Comité podrá invitar a los organismos especializados y órganos de las Naciones Unidas, así como a las organizaciones intergubernamentales y demás órganos interesados, a que presenten, para su examen por el Comité, información escrita respecto de las cuestiones tratadas en la presente Convención que caigan dentro del ámbito de sus actividades." (Naciones Unidas, 1990).

${ }^{33} \mathrm{El}$ artículo 30 del Reglamento del Comité CMW indica lo siguiente: "de conformidad con lo dispuesto en el artículo 74, párrafo 4, de la Convención, el Comité podrá invitar a los organismos especializados y órganos de las Naciones Unidas, así como a las organizaciones intergubernamentales y otros órganos interesados (entre ellos instituciones nacionales de derechos humanos, organizaciones no gubernamentales y otros órganos) a que le presenten, para su examen, información escrita respecto de las cuestiones tratadas en la Convención que estén comprendidas en 
De manera oficial, el Comité ha reconocido las aportaciones de las organizaciones de la sociedad civil, así como propuesto soluciones antes posibles limitantes financieras o logísticas por parte de éstas. El Comité ha resaltado las siguientes funciones por parte de las ONG: (1) consultas y aportaciones al informe del Estado parte; (2) informes alternativos y presentación de la información oral en las sesiones del Comité; (3) aportaciones a las observaciones generales del Comité y utilización de las mismas; (4) apoyo en cuestiones de comunicaciones individuales - una vez que esta facultad entre en vigor; (5) actividades de difusión a nivel global (Comité CMW, 2014). No obstante, esta relación no siempre fue así. La sociedad civil fue poco participativa en el proceso de elaboración de la Convención. Esta situación fue sorpresiva, debido principalmente a que ya se había documentado la participación de las ONG en la redacción de otros instrumentos dentro del Sistema Universal de Derechos Humanos, como la CEDAW o la CAT (Simeone \& Piper, 2017, p. 61). Como se ha documentado, hubo el intento por parte de delegaciones de establecer un contacto con distintas ONG en el proceso, lo cual representa una participación mínima (Hune, 1987).

El escenario internacional propio de la Guerra Fría hacía que el interés primario de estuviera enfocado en otros temas, antes que las necesidades específicas de la población migrante, sobre todo ante la división Norte-Sur que representaban las discusiones en los trabajos preparatorios de la Convención (Simeone \& Piper, 2017, pp. 61-62). Asimismo, dentro de los países que lideraban el proyecto, o no había el interés dentro de la sociedad civil de participar o no se contaba con la suficiente presión. Finalmente, el hecho que las negociaciones tuvieran lugar principalmente en Nueva York dificultaba el traslado de diversas ONG, sobre todo de aquéllas que ya estaban acostumbradas a tener una actividad en Ginebra (Grange \& D'auchamp, 2009, pp. 71-74).

A pesar de ello, diversos autores reconocen que la sociedad civil tuvo un papel catalizador para la promoción de la ratificación de la CMW. Aunado al aumento de ONG dedicadas a la lucha por los derechos humanos al final de la Guerra Fría, el impulso de la promoción de la CMW por parte de la sociedad civil fue particularmente relevante debido a que no había alguna agencia internacional que tomara esta iniciativa (Taran \& Niessen, 1991, p. 859; citado en Simeone \& Piper, 2017, p. 63). Esto no quiere decir que fuera tarea fácil, sobre todo considerando que no había voluntad por parte de los países occidentales para ratificar

el ámbito de sus actividades." (Comité CMW, 2019). También el Comité ha publicado directrices sobre la presentación de información alterna por parte de agentes de la sociedad civil (Comité de Trabajadores Migratorios, 2018). 
este instrumento, lo cual hizo que el ejercicio de promoción fuera uno en solitario (Grange \& D'auchamp, 2009, pp. 76-78).

Se han llevado a cabo diversas estrategias encaminadas a promover la ratificación. La campaña más importante a nivel mundial fue la Campaña Global por la Ratificación de la CMW, esfuerzo iniciado en 1998 por el Comité Directivo de la Campaña Mundial para la Ratificación de la Convención sobre los Derechos de los Trabajadores Migratorios para acelerar los procesos de ratificación y la entrada en vigor de la Convención. También se han convocado campañas internacionales de concientización, como la que se lanzó en 2015 para conmemorar el $25^{\circ}$ aniversario de la adopción de la CMW, organizada por el Foro de Migración en $\mathrm{Asia}^{34}$. A nivel nacional, también se han llevado a cabo, sin tanto éxito, campañas de ratificación, como por ejemplo en Canadá, Alemania, Gran Bretaña (De Guchteneire \& Pécoud, 2009, pp. 28-29). Finalmente, la sociedad civil ha promovido la Convención colaborando otros foros internacionales y en otras agencias de la ONU.

Asimismo, la sociedad civil colabora activamente en las labores del Comité. Como se sabe, la participación más "tradicional" por parte de las ONG en los Comités es la de la entrega de información alterna a los informes periódicos de los Estados. A la fecha, se tienen contabilizados 152 informes por parte de las organizaciones de la sociedad civil, la mayoría de ellos (126) corresponden a informes alternos o informes sombra (OACNUDH, 2019).

Aunque los datos recabados por Naciones Unidas merecen un análisis más profundo sobre la estructura y procesos para la entrega de informes sombra en los exámenes de los Estados parte, se contabilizan treinta países de los que el Comité ha recibido información alterna. En este ejercicio, sobresalen los informes entregados en los casos de México (25 informes sombra), Argentina (13), Chile (11) e Sri Lanka (10). Esto coincide con el número de exámenes periódicos a los que se han sometido los países (por ejemplo, México ha entregado tres informes), aunque también puede coincidir con la manera en que las organizaciones locales interactúan, en general, con otros comités.

\footnotetext{
${ }^{34}$ Esta campaña tiene como objetivos: (1) promover la ratificación de la CMW; (2) concientizar sobre la detención de menores migrantes; (3) concientizar sobre la contribución de trabajadores migrantes domésticos; y (4) alertar sobre situaciones de trabajos forzados, trata de personas y prácticas de esclavitud. Para los eventos de esta campaña, puede consultarse (Migration Forum in Asia, 2019).
} 
De los países que son parte de la Convención cuando el Comité inició sus sesiones, nueve no tienen registrados informes sombra ${ }^{35}$. Indonesia figura como el único país con una ratificación reciente (2012) que tiene un número considerable de este tipo de documentos (OACNUDH, 2019). Por lo tanto, también se puede deducir que no hay una práctica constante en la entrega de informes sombra en este Comité. El Comité ha intentado promover la participación de este sector en la presentación de los informes, como pasó con el informe inicial de Mali (Comité CMW, 2006) e, incluso, el Comité se ha pronunciado cuando no observa participación de la sociedad civil, como pasó en el caso del segundo informe periódico de Ecuador (Comité CMW, 2011).

Se observa que los informes sombra entregados se generan de manera colaborativa, a través de redes entre ONG. De acuerdo con los datos presentados por la OACNUDH (2019), son pocos los informes alternos en donde figure alguna organización en solitario. Asimismo, el Comité ha adoptado medidas para la presentación de informes alternos por parte de las ONG, tales como dedicar algún tiempo durante sus sesiones para la presentación de estos o aprovechar herramientas, como las videoconferencias (Comité CMW, 2014).

El Comité ha sostenido, desde el inicio de sus actividades, distintas reuniones con organizaciones de la sociedad civil para fomentar la ratificación y puesta en práctica de la Convención ${ }^{36}$, o para celebrar debates generales, siendo estas últimas importantes para la elaboración de observaciones generales ${ }^{37}$. Finalmente, tanto

${ }^{35}$ Estos países son: Bosnia Herzegovina, Cabo Verde, Ghana, Guinea, Lesoto, Perú, Seychelles, Siria y Timor-Leste.

36 Por ejemplo, en 2015 se llevaron a cabo diversos eventos en Ginebra y en Nueva York para conmemorar el $25^{\circ}$ aniversario de la adopción de la Convención (Comité CMW, 2016). También, durante 2016 y 2017 se llevaron a cabo talleres de capacitación y sensibilización sobre el contenido de la Convención (Comité CMW, 2017).

${ }^{37}$ En octubre de 2009, el Comité organizó un debate general sobre los trabajadores domésticos migratorios, en la cual asistieron diversas ONG provenientes de Bahréin, Israel, Italia, Líbano, Países Bajos y Senegal, así como representantes de redes regionales e internacionales, además que otros grupos de la sociedad civil mandaron información por escrito (Camerún, Costa Rica, El Salvador, Filipinas, Malasia y Nigeria (Comité CMW, 2010). En septiembre de 2011 se llevó a cabo un debate general sobre los derechos de los trabajadores migratorios en situación irregular y sus familiares (Comité CMW, 2012). También se han organizado debates generales sobre la niñez migrante, en conjunto con el Comité de los Derechos del Niño (Comité CMW, 2016) (Comité CMW, 2017) (Comité CMW, 2018).

A partir de 2013, el Comité celebró con más frecuencias debates generales. En abril de 2013, se celebró un debate general sobre la importancia de las estadísticas de migración para la pre- 
la sociedad civil como los expertos del Comité han coincidido en otros foros, tal como las ediciones del Foro Mundial sobre Migración y Desarrollo (Comité CMW, 2012) (Comité CMW, 2015) (Comité CMW, 2016) o en eventos organizados por el Consejo de Derechos Humanos (Comité CMW, 2016).

Esta cooperación tiene ventajas, tanto para el Comité, como para las organizaciones de la sociedad civil. De acuerdo con Grange y D'auchamp, (2009, p. 83), estas redes han podido monitorear la adopción de política migratorias acordes con los documentos adoptados en estas conferencias. Por parte del Comité, es posible la difusión de la información de lo que hace el Comité por parte de las ONG, sobre todo tomando en cuenta que no todas las organizaciones tienen la misma capacidad de acceder a las actividades que lleva a cabo el Comité38.

Más allá de la cooperación con el Comité, la sociedad civil ha encontrado otras estrategias que permiten una mayor visibilidad sobre los estándares de protección de la comunidad migrante, que van desde la incorporación de información alterna en otros mecanismos convencionales y no convencionales de la ONU, hasta la participación en otros foros internacionales. Por ejemplo, la sociedad civil se ha involucrado con el Relator Especial sobre los Derechos Humanos de los Migrantes, quien también ha cooperado en diversas ocasiones con el Comité. Asimismo, la práctica de participación de las ONG en los distintos comités es recurrente, además que estos han llevado a cabo interpretaciones de estándares aplicables a la población migrante en sus recomendaciones generales y observaciones por país $^{39}$. Aunque este ejercicio podría mejorar ante la ratificación de la CMW y una mejor coordinación entre los mismos comités, resulta interesante esta práctica que también ha tratado de monitorear el mismo Comitée ${ }^{40}$.

sentación de informes, donde también participaron distintas ONG (Comité CMW, 2013). En abril de 2014, se celebró el debate general sobre la protección y explotación en el lugar de trabajo (Comité CMW, 2014).

${ }_{38}$ Un ejemplo de la manera en que el Comité y las ONG forman redes de colaboración es a través del Comité Directivo de la Campaña Mundial para la Ratificación de la Convención sobre los Derechos de los Trabajadores Migratorios. Algunos de los logros de esta red se encuentran: (1) publicaciones que sirven de guías para nuevas ONG que quieran trabajar con el Comité; y (2) asesoría en la elaboración de informes sombra ante el Comité (Grange \& D'AUCHAMP, 2009, pp. 89-91).

${ }^{39}$ Por ejemplo, WEISSBRODT \& RHODES (2013, pp. 71-111) hacen un estudio sobre la protección de los derechos de los trabajadores migrantes en las observaciones finales y las recomendaciones generales adoptadas por diversos comités para países de Medio Oriente.

${ }^{40}$ En su segundo informe anual, el Comité CMW destacó la reunión que sostuvo con December 18 y 
Otra estrategia ha sido la colaboración de las ONG en otros foros internacionales sobre el tema migratorio. Sin embargo, éstas han tenido que reformular sus objetivos ante el continuo desinterés por ratificar la CMW. Por ejemplo, se ha capitalizado la oportunidad de participar en foros regionales e internacionales como el Foro Mundial de Migración y Desarrollo (Grange \& D'auchamp, 2009, pp. 92-95), además que se han creado redes de colaboración, incluso con el sector académico (Piper, 2015, pp. 797-798). El esfuerzo más reciente fue la participación de la sociedad civil en los procesos de consulta para la adopción del Pacto Mundial para la Migración ${ }^{41}$. Por lo tanto, las organizaciones dedicadas a la promoción de los derechos de la comunidad migrante se enfocan cada vez con menos prioridad en la promoción de la ratificación de la CMW como tal.

Autores más optimistas están poniendo atención al Examen Periódico Universal del Consejo de Derechos Humanos (EPU), al ser un mecanismo más visible que los mecanismos convencionales. En un estudio realizado sobre las recomendaciones relacionadas con la CMW a los países de la Unión Europea, Alan Desmond (2015, pp. 60-67) concluyó que el EPU puede ser un mecanismo que catalice nuevos debates, no sólo en torno a la ratificación de la $\mathrm{CMW}$, sino también cierta "vinculación" con el objetivo principal de la Convención. Este mecanismo podría compensar la falta de consenso de la CMW, siempre y cuando haya una congruencia en la interpretación de los principios de protección de la población migrante.

la Comisión Católica Internacional de Migración para que elaboraran un estudio sobre cómo otros comités tratan el tema migratorio en sus observaciones finales (Comité CMW, 2005).

${ }^{41}$ Los procesos de consulta fueron regionales y se llevaron a lo largo de 2017 en Bangkok, Tailandia (organizado por Migrant Forum in Asia), Beirut, Líbano (organizado por Cross-Regional Center for Refugees and Migrants), Bamako, Mali (organizado por Pan-African Network in Defense of Migrants Rights y MADE- Afrique), Washington, D.C., Estados Unidos (organizado por AFL-CIO, Alianza Americas, National Network for Immigrant and Refugee Rights, y Solidarity Center), Bruselas, Bélgica (organizado por Migration and Development Civil Society Network y Platform for the International Cooperation on Undocumented Migrants), Quito, Ecuador (organizado por Bloque Latinomericano de Asylum Access Ecuador) y Nadi, Fiji (organizado por Pacific Islands Association of Non-Governmental Organisation, Fiji Citizens Constitutional Forum, Sydney Asia Pacific Migration Center (Universidad de Sidney) Diplomacy Training y Migrant Forum Asia) (Naciones Unidas, 2019). Cabe destacar que muchas de estas organizaciones forman parte de una red mayor, Global Coalition on Migration, lo cual ayudó a clarificar la aportación de la sociedad civil en el Pacto Mundial. Cabe destacar que si bien uno de los objetivos es llamar a la ratificación de la CMW, se establecen otros objetivos concisos para el respeto de los derechos humanos de los migrantes (Global Coalition for Migration, 2017). 


\section{Conclusiones}

El objetivo general de este trabajo de investigación fue evaluar el impacto de las acciones de la sociedad civil en el ámbito internacional de derechos humanos como estrategia de presión hacia los Estados. Se analizó si el consenso internacional de una norma de derechos humanos es o no una limitante en el campo de acción de la sociedad social como estrategia de presión hacia los Estados

Un ejemplo en donde se observa una falta de consenso internacional es la Convención para la Protección de todos los Trabajadores Migratorios y sus Familias. Por lo tanto, se hizo un breve análisis sobre el impacto que esta situación tiene en la generación de estrategias de presión por parte de la sociedad civil.

Si bien se puede concluir que la CMW es el primer instrumento jurídico en establecer un esquema integral de protección a los trabajadores y sus familiares - cualquiera que sea su estatus legal, también es cierto que es un tratado que ha sido objeto de un gran desacuerdo internacional en cuanto a la gobernabilidad migratoria. Esta situación puede observase, no sólo con el bajo número de ratificaciones, sino por el continúo desinterés en la comunidad internacional por promover de manera activa el contenido de este instrumento.

Por lo tanto, ¿la falta de consenso internacional sobre la CMW significa que la sociedad civil no tome como instrumento de reivindicación? ¿En qué medida existe la voluntad de cooperar con el Comité para generar presión a los Estados que sí son parte? En primer lugar, si bien la sociedad civil no se mostró totalmente participativa en la elaboración de este instrumento, sí ha sido reconocido su papel como catalizador para la ratificación de este instrumento, sobre todo para que lograra entrar en vigor en 2003.

En una revisión sobre la interacción de la sociedad civil y el Comité, se puede observar que existe cierto desinterés, si solamente tomamos en cuenta la baja participación de la sociedad civil en la revisión de los informes periódicos de los Estados Parte. Sin embargo, también hay que considerar que muchas de estas organizaciones actualmente actúan en redes transnacionales y la manera de interactuar con el Comité ha ido más allá de los exámenes de los informes de los Estados: se podría decir que se encuentra en el interés de las ONG dar una mayor visibilidad a temas que han aparecido en el nuevo contexto migratorio a nivel mundial.

Ante un débil consenso de la CMW, otras estrategias han sido exploradas. Por ejemplo, las ONG continúan participando en otros mecanismos convencionales, 
por lo que interpretaciones sobre contenidos similares a la Convención pueden salir a la luz. Asimismo, ha aumentado el interés por participar en mecanismos como el Examen Periódico Universal del Consejo de Derechos Humanos.

Sin embargo, la estrategia de la sociedad civil en la promoción de los derechos de los migrantes va más allá, no sólo del Comité, sino de todo el Sistema Universal de Derechos Humanos. Este capítulo hizo un recuento sobre algunos foros internacionales en los que la sociedad civil ha formado parte, siendo el ejemplo más reciente su participación para la adopción del Pacto Global por la Migración. En estos esfuerzos la promoción de la ratificación de la Convención se menciona, pero en un segundo plano.

Como se puede observar, la falta de consenso internacional de la CMW ha constituido un reto para la sociedad civil en su lucha por la reivindicación de los derechos de los trabajadores migrantes. Sin embargo, el Comité sigue siendo percibido como un mecanismo que puede ayudar a consolidar la lucha, sobre todo en estos tiempos donde el fenómeno migratorio se ha complicado aún más.

\section{Bibliografía}

Asamblea General de Naciones Unidas (2014), Aplicación de los instrumentos de derechos humanos. Nueva York: Naciones Unidas.

BATTISTELLA, G. (2009), "Migration and human rights: the uneasy but essential relationship", en R. Cholewinski, P. De GuChteneire y A. Pécoud, Migration and human rights: the United Nations Convention on Migrant Workers (pp. 47-69), Cambridge: Cambridge University Press.

Bustamante, J. (2002), "'Immigrants' vulnerability as subjects of human rights". The International Migration Review, 333-354.

CAMPESI, G. (Septiembre de 2012), "Migraciones, seguridad y confines en la teoría social contemporánea". (U. d. Barcelona, Ed.) Revista Crítica Penaly Poder(e), 1-20.

Canales, A. (2015), E pur si muove: Elementos para una teoría de las migraciones en el capitalismo global. México: Porrúa.

Cano, M. (2010), "Protección internacional de los derechos humanos de los trabajadores migratorios". Persona y derecho, 137-162. 
Carrera, S., Lannoo, K., Stefan, M. y Vosylüté, L. (Noviembre de 2018), "Some EU governments leaving the UN Global Compact on Migration: a contradiction in terms?" Policy Insights (2018-15).

Comité CMW (2005), Informe del Comité de Protección de los Derechos de todos los Trabajadores Migrantes y de sus Familiares - Segundo periodo de sesiones. Ginebra: Naciones Unidas.

- (2006), Informe del Comité de Protección de los Derechos de todos los Trabajadores Migrantes y de sus Familiares - tercer y cuarto periodo de sesiones. Ginebra: Naciones Unidas.

- (22 de mayo de 2008), Guidelines for the periodic reports to be submitted by states parties under Article 73 of the Convention. Ginebra.

- (2010), Informe del Comité de Protección de los Derechos de todos los Trabajadores Migrantes y de sus Familiares $-11^{\circ}$ y $12^{\circ}$ periodo de sesiones. Ginebra: Naciones Unidas.

- (2011), Informe del Comité de Protección de los Derechos de todos los Trabajadores Migrantes y de sus Familiares $-13^{\circ}$ y $14^{\circ}$ periodo de sesiones. Ginebra: Naciones Unidas.

- (2012), Informe del Comité de Protección de los Derechos de todos los Trabajadores Migrantes y de sus Familiares $-15^{\circ}$ y $16^{\circ}$ periodos de sesiones. Ginebra: Naciones Unidas.

- (2013), Informe del Comité de Protección de los Derechos de todos los Trabajadores Migrantes y de sus Familiares $-18^{\circ}$ y $17^{\circ}$ periodo de sesiones. Ginebra: Naciones Unidas.

- (2014), "Declaración del Comité de Protección de los Derechos de Todos los Trabajadores Migratorios y de sus Familiares sobre su relación con las organizaciones de la sociedad civil, aprobada en su $21^{\circ}$ período de sesiones ( 1 a 5 de septiembre de 2014)", CMW/C/21/2. Ginebra: Naciones Unidas.

- (2014), Informe del Comité de Protección de los Derechos de todos los Trabajadores Migrantes y de sus Familiares $-19^{\circ}$ y $20^{\circ}$ periodo de sesiones. Ginebra: Naciones Unidas. 
- (2015), Informe del Comité de Protección de los Derechos de todos los Trabajadores Migrantes y de sus Familiares $-21^{\circ}$ y $22^{\circ}$ periodos de sesiones. Ginebra: Naciones Unidas.

- (2016), Informe del Comité de Protección de los Derechos de todos los Trabajadores Migrantes y de sus Familiares $-23^{\circ}$ y $24^{\circ}$ periodos de sesiones. Ginebra: Naciones Unidas.

- (2017), Informe del Comité de Protección de los Derechos de todos los Trabajadores Migrantes y de sus Familiares $-25^{\circ}$ y $26^{\circ}$ periodos de sesiones. Ginebra: Naciones Unidas.

- (7 de septiembre de 2017), Input for the Secretary-General's Report on the Global Compact on Migration. Obtenido de hitps://tbinternet.ohchr.org/Treaties/ CMW/Shared\%20Documents/1_Global/INT_C MW_INF_8293_E.pdf.

- (2018), Contribution to the 2030 Sustainable Development Goals in response to a call for inputs by the High-Level Political Forum on Sustainable Development (HLPF), Ginebra: Naciones Unidas.

- (2018), Informe del Comité de Protección de los Derechos de todos los Trabajadores Migrantes y de sus Familiares $-27^{\circ}$ y $28^{\circ}$ periodos de sesiones. Nueva York: Naciones Unidas.

- (2019), "Reglamento". CMW/C/2. Ginebra: Naciones Unidas.

Comité de Trabajadores Migratorios (2018), "Directrices para la presentación de informes por los organismos especializados y otros órganos del Sistema de las Naciones Unidas". CMW/C/1. Naciones Unidas.

De GuChteneire, P. y PéCoud, A. (2009), "Introduction: the UN Convention on Migrant Rights", en R. ChOlewinskI, P. De GuChteneire y A. Pécoud, Migration and human rights: the United Nations Convention on Migrant Workers' Rights (pp. 1-44), Cambridge: Cambridge University Press.

- (2008), "La Convención de las Naciones Unidas sobre los Derechos de los Trabajadores Migrantes: obstáculos, oportunidades y perspectivas". Migraciones, 9-55.

DESMOND, A. (2015), "The triangle that could square the circle? The UN International Convention on the Protection of the Rights of All Migrant Workers and Members 
of their Families, the EU and the Universal Periodic Review". European Journal of Migration, 39-69.

EdelenbOS, C. (2009), "Committee on Migrant Workers and implementation of the ICRMW", en R. CholeWinski, P. De GuChteneire y A. PéCOUd, Migration and human rights: The United Nations Convention on Migrant Workers' Rights (pp. 100-121), Cambridge: Cambridge University Press.

Georgopoulou, A., SchrempF, T., y Venturi, D. (2017), "The added value of the ICRMW's substantive provisions", en A. DESMOND, Shining new light on the UN Migrant Workers Convention (pp. 129-150), Pretoria: Pretoria University Law Press.

Global Coaltion for Migration (5 de mayo de 2017), Key issues for the Global Compact. Obtenido de http://gcmigration.org/wp-content/uploads/2017/05/ May-2017-GCM- GC-Key-issues-Rev.pdf.

Grange, M. (2017), "Safeguarding migrants against arbitrary detention", en A. DESMOND, Shining new light on the UN Migran Workers Convention (pp. 151-175), Pretoria: Pretoria University Law Press.

Grange, M. y D'AuChamp, M. (2009), "Role of civil society in campaigning for and using the ICRMW", en R. CholewinskI, P. De Guchteneire y A. Pécoud, Migration and human rights: the United Nations Convention on Migrant Workers' Rights (pp. 70- 99), Cambridge: Cambridge University Press.

GRANT, S. y LYON, B. (2017), "Impact and use of the ICRMW in other UN fora", en A. DESMOND, Shining new light on the UN Migrant Workers Convention (pp. 101- 128), Pretoria: Pretoria University Law Press.

HUNE, S. (1987), "Drafting an International Convention on the Protection of the Rights of All Migrant Workers and Their Families". The International Migration Review, 123-127.

MARTIN, S. y ABIMOURChED, R. (2009), "Migrant Rights: international law and national action. International Migration, 115-138.

Migrant Forum In Asia (1 8 de agosto de 2019), CMW timeline. Obtenido de hittp:// cmw25.org/cmw-timeline/

- (29 de agosto de 2019), Step it up: dignity, rights, development - marking 25 years of the UN Migrant Workers Convention. Obtenido de http://cmw25.org/. 
NACIONES UNIDAS (18 de Diciembre de 1990), "Convención internacional sobre la protección de los trabajadores migratorios y de sus familiares". Nueva York.

- (2005), Compilación de directrices relativas a a forma y el contenido de los informes que deben presentar los Estados partes en los tratados internacionales de derechos humanos. Ginebra.

- (2017), Making migration work for all. Informe del Secretario General, Naciones Unidas, Nueva York.

- (1 1 de Diciembre de 2018), Pacto Mundial para la Migración Segura, Ordenada y Regular. Obtenido de https://undocs.org/es/A/CONF.231/3.

- (2018), Status of the human rights treaty body system. Nueva York: Naciones Unidas.

- (2018), Status of the human rights treaty body system: Addendum. Nueva York: Naciones Unidas.

- (31 de agosto de 2019), Compact forMigration: regional civil society consultations. Obtenido de https://refugeesmigrants.un.org/regional-civil-society-consultations.

Nafziger, J. y Bartel, B. (1991), "The Migrant Workers Convention: Its Place in Human Rights Law". The International Migration Review, 771-799.

OACNUDH. (2006), La Convención internacional sobre trabajadores migratorios y su comité. Ginebra: Naciones Unidas.

- (18 de diciembre de 2018), Los Estados deben garantizar a todos los migrantes gozan de todos los derechos humanos, expertos de la ONU dicen sobre el Día Internacional del Migrante. Obtenido de https://www.ohchr.org/Documents/ HRBodies/CMW/StatementCMW_SRMigrants_18Dec2018.docx.

- (27 de agosto de 2019), UN treaty body database. Obtenido de https://tbinternet.ohchr.org/SitePages/Home.aspx.

- (30 de agosto de 2019), UN Treaty Body Database. CMW: information from Civil Society Organizations. Obtenido de http://tbinternet.ohchr.org/_layouts/15/ treatybodyexternal/TBSearch.aspx? Lang=en\&TreatyID=7\&DocTypelD=14\&DocTy pelD $=141$ \&DocTypelD=139\&DocTypel $\mathrm{D}=145 \&$ DocTypeCategoryII $=4 \&$ DocType CategoryID=1. 
OrganizaCión InternaCIONAL del Trabajo (18 de agosto de 2019), NORMLEX: Information System on International Labour Standards. Obtenido de https://www.ilo.org/ dyn/normlex/es/f?p=NORMLEXPUB:12030:0::NO:::\#Trabajad ores_migrantes.

Organización Internacional para las Migraciones (2018), World Migration Report 2018. Obtenido de https://www.iom.int/wmr/world-migration-report-2018.

PÉCOUD, A. (2017), "The politics of the UN Migrant Workers Convention", en A. DESMOND, Shining new light on the UN Migrant Workers Convention (pp. 24-44), Pretoria: Pretoria University Law Press.

PIPER, N. (2015), "Democratising migration from the bottom up: the rise of the global migrant rights movement". Globalizations, 788-802.

RODERA RANZ, S. (2014), La protección internacional de la persona migrante en situación irregular: estudio de la Convención Internacional sobre la Protección de los Derechos de todos los Trabajadores Migratorios y de sus Familiares. Zaragoza: Universidad de Zaragoza.

RUHS, M. (2013), The price of rights: regulating international labor migration. Princeton: Princeton University Press.

SIMEONE, L. y PIPER, N. (2017), "Making rights in times of crisis: civil society", en A. DESMOND, Shining new light on the UN Migrant Workers Convention and the Migrant Workers Convention (pp. 45-71), Pretoria: Pretoria University Law Press.

SJÖBlO, M. SCHIFF, M. y MORRISON, A. (2008), The international migration of women. Obtenido de hitp://documents.worldbank.org/curated/en/673051468328175051/ pdf/417880PAPEROMi101OFFICIALOUSEOONLY1.pdf.

SLINCKX, I. (2009), "Migrants' rights in UN human rights conventions", en R. CHOLEWINSKI, P. De GuChtenelRe, y A. PÉcoud, Migration and Human Rights: The United Nations Convention on Migrant Workers' Rights (pp. 122-149), Cambridge: Cambridge University Press.

Slocum, J. (2017), "Migration and refugees at the United Nations. Toward new Global Compacts and the emergence of an international migration regime". Notes Internationals CIDOB.

Steering Committee on Global Campalgn for Ratification of the Convention on the RightS OF Migrants (29 de agosto de 2019), Global Campaign for Ratification of 
the Convention on the Rights of Migrants. Obtenido de https://migrantsrights. org/campaign.htm.

Taran, P. (2000), "Status and prospects for the UN Convention on Migrants' Rights". European Journal of Migration and Law, 85-100.

Taran, P. y Niessen, J. (1991), "Using the new Migrant Workers' Rights Convention". International Migration Review, 859-865.

Unión Interparlamentaria, Organización InternaCiOnal del Trabajo y OACNUDH (2015), Migration, human rights and governance: handbook for parliamentarians no. 24. Ginebra: Naciones Unidas.

United Nations Treaty Collection (24 de agosto de 2019), Status of treaties: International Convention on the Protection of the Rights of All Migrant Workers and Members of their Families. Obtenido de https://treaties.un.org/Pages/ViewDetails. aspx? src=TREATY\&mtdsg_no=IV-13\&chapter=4\&clang=_en.

UNODC (25 de agosto de 2019), United Nations Convention against Transnational Organized Crime and the Protocols thereto. Obtenido de https://www.unodc.org/ unodc/en/organized-crime/intro/UNTOC.html.

WeISSBRODT, D. y RHODES, J. (2013), "United Nations Treaty Body monitoring of migrant workers in the Middle East". Middle East Law and Governance, 71-111.

WeXLER, L. (2007), "The non legal role of International Human Rights Law in addressing immigration". University of Chicago Legal Forum, 359-403. 